\title{
Theileria survival and the spindle
}

A new paper in PLoS Biology reports how the apicomplexan parasite Theileria annulata usurps the host cell mitotic apparatus to ensure parasite survival and persistence.

Theileria spp. have a complex and fascinating life cycle. During the obligate intracellular schizont stage, the parasite is free in the host cell cytoplasm and forms a multinucleated syncytium that transforms parasitized cells, leading to continuous replication, resistance to apoptosis and a metastatic phenotype. It was known that the close association between Theileria spp. and elements of the host cell mitotic machinery was required for parasite segregation at cell division, but the exact mechanisms involved were unclear.

Conrad von Schubert and colleagues analysed the interaction between the T. annulata schizont and the host cell cytoskeleton and found that the schizont associates with newly formed astral and central spindle microtubules. This positions the parasite with the host chromosomes at the equatorial region of the cell during metaphase and at the cell midzone during anaphase.
Earlier work had shown that Theileria spp. can recruit host cell kinases to the parasite surface. Immunofluorescence in synchronized cultures was used to investigate whether any of the host cell kinases that are involved in mitosis associate with the T. annulata schizont. The authors found that Polo-like kinase 1 (PLK1), which is involved in a range of processes essential for mitosis, including central-spindle function and determining the position of

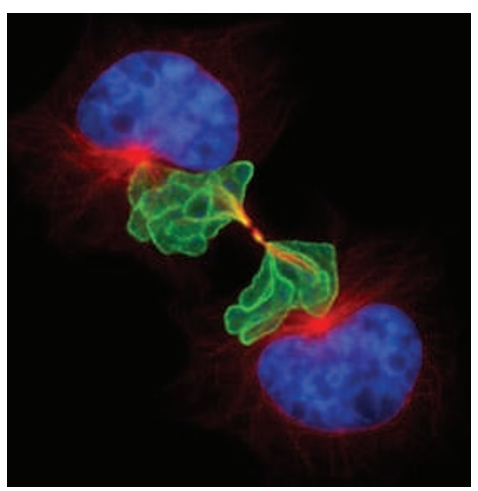

During cytokinesis, the Theileria annulata schizont (green) is included in the process of host cell abscission and distributed equally between the two daughter cells (nuclei are stained blue and microtubules are stained red). Image courtesy of D. Dobbelaere, University of Bern, Switzerland. the division site, is recruited to the schizont surface through its Polo box domain. PLK1 binding is negatively regulated by cyclin-dependent kinase 1 (CDK1) and varies with the progression of the cell cycle. PLK1 binds during G2, dissociates as the cells enter the early stages of mitosis and then re-associates at the onset of anaphase. Moreover, as the cells move into telophase, PLK1 associates with only those areas of the schizont surface that interact with the central spindle.

Further work demonstrated that PLK1 activity is required for the interaction between the schizont and the central spindle. Last, the authors provide direct evidence that the interaction between T. annulata and the astral and central spindle microtubules is required for equal schizont segregation during host cell division.

\section{Sheilagh Molloy}

ORIGINAL RESEARCH PAPER von Schubert, C. et al. The transforming parasite Theileria co-opts host cell mitotic and central spindles to persist in continuously dividing cells. PLoS Biol. 8 , e1000499 (2010) 